\title{
PDMS o UDMS. Dos perspectivas de gestión clínica en Medicina Intensiva
}

\author{
R. ABIZANDA CAMPOS, A. FERRÁNDIZ SELLÉS Y R. REIG VALERO \\ Servei de Medicina Intensiva. Hospital Universitario Asociado General de Castelló. \\ Castelló de la Plana. Castellón. España.
}

La necesidad de disponer de la máxima información posible que traduzca el trabajo realizado en las Unidades de Cuidados Intensivos (UCI) tiene dos vertientes. Una centrada en la monitorización del paciente, los cambios registrados en su situación clínica y la respuesta que ellos condicionan en la actitud y acciones del equipo asistencial. La otra focalizada en la posibilidad de describir la actividad general de la U7nidad, las características epidemiológicas de la población atendida y los indicadores de efectividad y eficiencia que puedan traducirse en políticas de evaluación continua de calidad.

A la primera se le ha dado el nombre de control a nivel de paciente y a la segunda el de control a nivel de UCl.

La industria ha desarrollado instrumentos (informatizados) capaces de "capturar» la información procedente de los sistemas de monitorización para poder establecer la primera (los conocidos como patient data management systems [PDMS]), pero desafortunadamente no ha solventado el salto informativo del paciente individual a la descripción global de la actividad.

Los autores defienden la necesidad de disponer de los unit data management systems (UDMS), carencia no bien resuelta, como complemento imprescindible de la información en tiempo real procedente de los pacientes.

Correspondencia: Dr. R. Abizanda.

Servei de Medicina Intensiva.

Secretaría de la Unidad de Cuidados Intensivos.

Hospital Universitario Asociado General de Castelló.

Avda. Benicàssim, s/n.

12004 Castelló de la Plana. Castellón. España.

Correo electrónico: abizanda_ric@gva.es rabizandac@terra.es

Manuscrito aceptado el 30-VII-2007.
PALABRAS CLAVE: PDMS (patient data management system), UDMS (unit data management system), gestión clínica, sistemas de gestión de paciente, sistemas de gestión de UCI.

\section{PATIENT DATA MANAGEMENT SYSTEMS OR UNIT DATA MANAGEMENT SYSTEMS. TWO CLINICAL MANAGEMENT PERSPECTIVES IN INTENSIVE MEDICINE}

The need of availability of information able to describe the activity performed in ICU has two different sides. The first related with the monitoring of the patient himself, his clinical situation changes and the cehecking of attitudes and reactions of the clinical team related to these changes. The other one focused on the possibility to describe appropriately the general activity of the unit, the epidemiological characteristics of the attended population and the indicators of efectitivity and efficiency that could be used for a continous quality improving.

The first one has been named as patient level control, and the second one as unit level control.

Industry tried to develop potent instruments (informatized) able to "capture" information from patient monitoring systems in order to cope with the first quoted need (the so called patient data management systems [PDMS]), but has not yet be able to cross the line between the individual patient and the global unit activity.

Authors emphasize the need of having the unit data management systems (UDMS), a not solved problem, as a complement on "real time on line" information obtained from patients.

KEY WORDS: PDMS (patient data management system), UDMS (unit data management system), clinical management. 


\section{INTRODUCCIÓN}

Durante el Congreso Nacional de la Sociedad Española de Medicina Interna Crítica y Unidades Coronarias (SEMICYUC), celebrado en Madrid, los días 4 a 6 de junio de 2007, tuvo lugar un simposio sobre sistemas informatizados de gestión en Medicina Intensiva en el que tuve la oportunidad de intervenir, estructurando las conclusiones a las diferentes ponencias de los oradores programados.

Debo reconocer que no supe evitar la tentación de plantear múltiples dudas que me asaltan personalmente al considerar el fondo de la cuestión tratada. Ante todo quiero dejar claro que en la Unidad que dirijo no se dispone de un sistema informatizado de gestión de pacientes (patient data management system [PDMS]) $\mathrm{y}$, sin embargo, creo que puedo afirmar que, dentro de nuestra especialidad, es conocida mi preocupación por disponer de la máxima información posible que permita establecer la gestión clínica del Servicio.

Sin duda, la estructura sanitaria, nuestro propio centro, dispone de sistemas de información genéricos que deben estar enfocados a la macrogestión, a la gestión de ese centro. Y como flecos colgantes, algunos de los datos que maneja esa información hacen referencia a indicadores de actividad del Servicio de Medicina Intensiva (SMI).

¿Se puede con ello establecer las líneas de gestión de ese Servicio? ¿Puede esa información dar respuesta a las preguntas planteadas sobre cuál es el uso que se da a los recursos disponibles? ¿Se pueden tomar decisiones operativas para planificar la actividad futura a corto o medio plazo? Es decir, ¿se puede llevar a cabo una gestión clínica efectiva e intentar a la vez que sea eficiente? Yo creo que no.

¿Dónde está entonces la solución? ¿Cómo debe generar el gestor clínico su propia información?

La respuesta ofertada en este momento se llama PDMS y estos sistemas permiten una adecuada gestión de paciente en el día a día ${ }^{1}$, sustituyen lo que una o más enfermeras, bien adiestradas, realizaban antes a pie de cama, permiten detectar errores de forma precoz y además permiten disponer de una cantidad ingente de datos, cuyo análisis proporciona una evidente mejora de la asistencia, puntual, individual, fundamental porque es la que justifica nuestra existencia como especialistas que forman un determinado colectivo. Lo primero, ante todo y pese a todo, el paciente. Siempre.

Desafortunadamente, estos sistemas no permiten llevar a cabo eso que hemos llamado gestión clínica. $¿$ La prueba?, hay excelentes profesionales asistenciales, con gran cantidad de conocimientos, habilidades, aptitudes, y hasta actitudes que deberían ser transmitidas como activo intangible, y que no son buenos gestores clínicos. Los árboles no les dejan ver el bosque. Todos nosotros podríamos poner, al menos, un nombre y dos apellidos a esta constatación.

\section{GESTOR Y GESTIÓN CLÍNICA}

Examinemos algunos conceptos ${ }^{2}$. Podemos definir gestión como el conjunto de reglas, métodos y procedi- mientos operativos para realizar con eficacia una actividad de empresa tendente a alcanzar objetivos concretos, gestionar como el hecho de tratar de alcanzar unos resultados de actividad con los recursos disponibles y un sistema de organización y gestor como el que gestiona siguiendo instrucciones y normativas recibidas.

Desde esta simple perspectiva, el gestor clínico trata de alcanzar unos resultados clínicos o asistenciales previstos, conforme a instrucciones u objetivos determinados, utilizando para ello unos recursos asistenciales que le son dados (equipamiento, personal, instalaciones, procedimientos de colaboración con otros servicios, etc.).

Dependiendo de que la organización asistencial responda a una titularidad pública o privada, las atribuciones e independencia, pero también la responsabilidad de las decisiones de gestión variarán según un amplio abanico de posibilidades ${ }^{3}$. Pero siempre las decisiones de gestión se tomarán en base a una información, la utilización de unos recursos y el objetivo de alcanzar unos determinados objetivos, conocidos por todos. El hecho que establece la diferencia entre la gestión clínica y otras formas de gestión es que sólo podemos realizarla los clínicos y ello es así porque muchas de las decisiones de gestión tienen componentes profesionales que sólo los clínicos podemos aportar, incluida la toma de decisiones en situación de incertidumbre ${ }^{4}$. Ahí está gran parte del meollo del problema: somos los clínicos, únicamente, los que podemos hacer aquello que se nos demanda y que nadie nos ha enseñado a hacer, sino en base a procesos de ensayo-error, una inmersión en conocimientos que nos son ajenos y que no nacen de la preeminencia del interés del paciente, sino de un ejercicio de gran esfuerzo y buena voluntad.

Por eso es tan fácil dejarnos sin argumentos. Tanto los directivos que dominan un lenguaje y unas técnicas que nos son casi desconocidas, como los proveedores (que intentan arrimar el ascua a su sardina atribuyendo a sus productos -tecnológicos o farmacológicos- unas posibilidades y beneficios casi mágicos). Y por el contrario, las necesidades básicas para nuestra labor, que frecuentemente identificamos con cambios en la dotación de personal, en las inversiones en instalaciones y en algunas otras «minucias» nos son permanentemente hurtadas a nuestra capacidad de decisión.

\section{CÓMO FUNCIONA}

Establecer una actividad de gestión clínica es utilizar unos recursos con un objetivo final predeterminado, para cuyo logro se precisan unos procedimientos intermedios, a la realización de los cuales se pueden establecer más o menos barreras. El esquema de la figura $1^{5}$ resume más claramente este mecanismo, y para que los distintos conceptos que incluyen los términos inputs, throughputs y outputs ${ }^{\mathrm{a}}$ queden suficientemen-

${ }^{\mathrm{a} C}$ Cabe recordar la diferencia entre output (resultado primario, por ejemplo mortalidad) y outcome (resultado final, por ejemplo reinserción social y laboral). 




Figura 1. Esquema genérico de funcionamiento de la gestión clínica (modificado de Spangenberg et $\mathrm{l}^{5}$ ).

te descritos, la tabla 1 presenta los conceptos que pueden incluirse bajo cada uno de estos epígrafes.

Es evidente que el examen superficial del esquema descrito en la figura 1 proporciona el máximo protagonismo a la «información», y si se nos plantease el tipo de información a que nos referimos, la respuesta debería ser: aquélla que permite modificar el distinto componente de esquema (inputs, throughputs, outputs y las barreras establecidas o existentes entre ellos).

A ello debemos añadir que el volumen de información que «maneja» una Unidad de Cuidados Intensivos (UCI) convencional es ingente ${ }^{6,7}$ y por ello mismo se hace preciso disponer de sistemas de captura de esa información que sean lo más adecuados posible y capaces de manejar todo ese volumen de datos.

\section{LA INFORMACIÓN. LO DISPONIBLE Y LO DESEABLE}

Al considerar los distintos tipos de información a los que puede recurrir el gestor clínico en Medicina
Intensiva, nos encontramos con una marcada ambivalencia que, como consecuencia de la presión de la industria, ha establecido límites o fronteras marcadamente dibujadas.

Un tipo de información hace referencia al control del paciente como tal (patient level management). La monitorización continua de los distintos parámetros fisiológicos y clínicos.

Son varios los programas disponibles comercialmente, habitualmente ligados a la línea de monitorización utilizada en el SMI en cuestión, que permiten un control exhaustivo de las variaciones de la situación clínica monitorizable del paciente, de su pauta terapéutica y de su modificación, y que además permiten recoger (si los sistemas electrónicos están interconectados) datos de los parámetros de ventilación, de los regímenes de trabajo de las bombas de infusión, de controles hemodinámicos o de dinámica intracerebral, e incluso de depuración extrarrenal en los casos en que esté establecida.

Se podría hablar del precio que se paga por este tipo de prestaciones, y no necesariamente de precio económico, sino de ratios de personal de enfermería, adiestramientos, caídas del sistema y otros aspectos puntuales, pero es evidente que este tipo de tecnología ha representado una importante mejora del seguimiento del paciente en el día a día y en las variaciones secuenciales. No solamente se ha incrementado la cantidad de datos disponibles sometidos al control de sistemas de alarma o aviso, sino que se han eliminado las variaciones interpersonales en la interpretación y detección de los cambios, y con ello la calidad asistencial ofertada es mucho mayor, y todo ello sin olvidarse de los inconvenientes que se han argumentado ante el uso generalizado de algunos indicadores incluidos $^{8,9}$.

No se tratarán aquí, ni siquiera superficialmente, las ventajas de disponer de una red hospitalaria, no sólo local del SMI, de registro y transmisión de la información, con la posibilidad de intercambio e interutilización de equipos en las distintas áreas asistenciales, porque de lo que se trata en este trabajo es de establecer valoraciones sobre la actividad intraservicio.

TABLA 1. Distintos componentes del proceso de gestión clínica incluidos en el esquema de la figura 1

\begin{tabular}{|c|c|c|c|}
\hline Input & \multicolumn{2}{|c|}{ Throughput } & Output \\
\hline Dimensionado & \multicolumn{2}{|l|}{ Protocolos asistenciales } & Análisis costo/beneficio \\
\hline Equipamiento/tecnología & \multicolumn{2}{|l|}{ Indicadores de actividad } & Análisis riesgo/beneficio \\
\hline Dotación humana & \multicolumn{2}{|l|}{ Cargas de trabajo } & Tasa de reingresos \\
\hline Nivel del hospital & \multicolumn{2}{|l|}{ Docencia } & Satisfacción del usuario \\
\hline Nivel de cuidados (nivel UCI) & \multicolumn{2}{|c|}{ Mortalidad predicha y real (comparación interna) } & Producción científica \\
\hline Cartera de servicios & & & Eficiencia e índices de eficiencia \\
\hline Case-mix & & & Calidad (comparación interna y respecto \\
\hline Sistema de organización & & & al estándar) \\
\hline Ventaja competitiva & & & Definición del producto estrella \\
\hline \multicolumn{2}{|c|}{ Barrera al input } & & Barrera al output \\
\hline \multicolumn{2}{|c|}{ Estrategias de ADT (criterio/indicación de ingreso, alta, triage) } & \multicolumn{2}{|c|}{ Identificación de servicio de destino } \\
\hline \multicolumn{2}{|c|}{ Circuitos } & \multirow{2}{*}{\multicolumn{2}{|c|}{$\begin{array}{l}\text { Control de la mortalidad post-UCI } \\
\text { Prestación outreach (UCI fuera de UCI) }\end{array}$}} \\
\hline \multicolumn{2}{|l|}{ Cultura del hospital } & & \\
\hline
\end{tabular}

UCI: Unidad de Cuidados Intensivos. 
El otro tipo de nivel que debemos considerar es el nivel del propio Servicio, o de la Unidad (si se prefiere considerarlo así): el unit level management. Veíamos en el apartado anterior cómo la información disponible y analizable sobre los inputs, los throughputs y las barreras a la entrada o salida de casos/pacientes del sistema condicionan los cambios secuenciales que se puedan establecer sobre los patrones de funcionamiento, el establecimiento de guías clínicas, la elaboración de recomendaciones de actuación y fundamentalmente el análisis pormenorizado de la actividad, con sus posibilidades de comparación tanto longitudinal (cambios en el tiempo del mismo SMI) como transversal (intercomparación de distintos SMI, distintos niveles hospitalarios, etc.).

Dicho de otra manera, el patient level management no permite conocer con el suficiente detalle aspectos de las prestaciones genéricas y colectivas de la asistencia ofertada. Y esta falta de información sólo puede suplirse con un enorme esfuerzo de personas dedicadas a la recogida de información y su análisis, que no se traduce en una mayor calidad de la asistencia proporcionada paciente a paciente, pero que es la que permite establecer las grandes líneas de actuación del Servicio (el unit level management). Como dice Nicolás Arcelís ${ }^{10}$ : «Es interesante que un sistema de información en UCI se acompañe de indicadores que evalúen el resultado del cuidado, que auditen el proceso asistencial y que permitan identificar oportunidades para una utilización más eficiente de los recursos. En este sentido, la incorporación de un cuadro de mandos integral puede facilitar la gestión de la UCI».

Lamentablemente, estas consideraciones no se han traducido en una disponibilidad estructurada y comercialmente asequible ${ }^{11}$. Ninguno de los sistemas de PDMS existentes proporciona (en nuestro conocimiento) la cantidad y calidad de datos requeridos. Existe una desafortunada desproporción entre lo deseable y lo disponible, que se acentúa en el momento en que se constata que la fuente de esta información que permite el unit level management es de distinta procedencia.

Una viene dada por el propio departamento de admisión del centro (los datos de características epidemiológicas y los que constituyen el conjunto mínimo básico de datos (CMBD) (mínimo requerible). Otra parte nace del análisis de los grupos uniformemente tratados en la Unidad (soporte ventilatorio, sepsis, control posquirúrgico urgente o programado, etc., sólo por citar algunos detalles). Y otra parte de los datos viene dada por la recogida, in situ, de aspectos no considerados habitualmente: aislamientos totales y por día, número de consultas extra Unidad con el tiempo requerido para su atención ${ }^{12,13}$ o cuantificación concreta de pacientes incorporados a ensayos clínicos o a programas de estudio concretos.

Algunos de estos ejemplos pueden ser extremadamente clarificadores: ¿existe algún sistema comercialmente disponible que cuantifique la limitación asistencial, transparente y pactada, asociada al rechazo de ingreso de un paciente en la UCI? ¿Puede alguien decir que está información es irrelevante a la

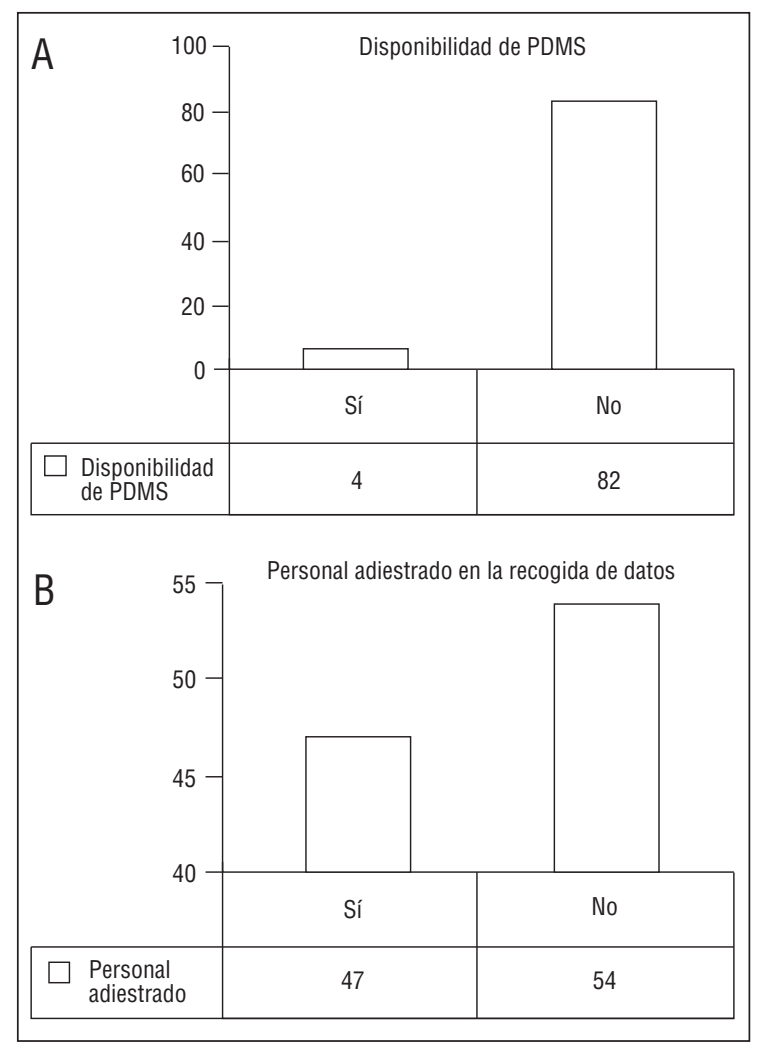

Figura 2. a) Porcentaje de Unidades de Cuidados Intensivos que disponen de patient data management systems (PDMS) (estudio SAPS), y dentro de ellas $b$ ) porcentaje de personal adiestrado para la recolección e interpretación de datos (datos procedentes del material suplementario electrónico del estudio SAPS 3, 2005 ${ }^{11}$ ).

hora de cuantificar la utilización de la dotación de camas del Servicio?

Seguro que no, y esta constatación sobre la limitada utilidad de los PDMS (y no sólo su precio) puede estar en la base de la muy escasa presencia de esta tecnología en las UCI de la Unión Europea (fig. 2), según datos proporcionados por un estudio multicéntrico harto conocido.

\section{A MODO DE ILUSTRACIÓN}

Que la utilidad de lo que hemos dado en llamar unit data management systems (UDMS) radica en permitir una gestión más adecuada de las propias responsabilidades asistenciales, puede verse ilustrada por los siguiente ejemplos prácticos sobre recogida e interpretación de la información disponible (si se quiere recoger y utilizar), con importantes repercusiones sobre el sistema de funcionamiento de un SMI polivalente de un hospital docente de referencia.

\section{Ejemplo A}

Tipo de los pacientes ingresados. Entre los años 2000 y 2005 (fig. 3) se observa que los pacientes in- 


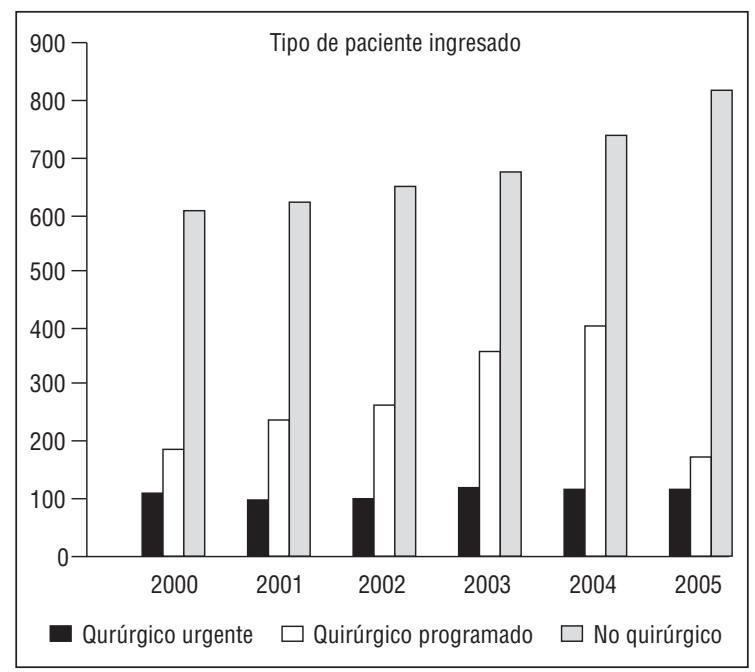

Figura 3. Evolución de los ingresos anuales conforme a la clasificación por tipo de paciente (quirúrgico urgente o programado y no quirúrgico) (datos propios).

gresados en nuestra UCI han representado un aumento progresivo del número de pacientes no quirúrgicos, un mantenimiento de los pacientes quirúrgicos urgentes y un salto decreciente (a partir de 2004) en los pacientes quirúrgicos programados.

El fenómeno observado tiene dos posibles explicaciones o, mejor, interpretaciones. Una, simple, es que la apertura de una REA, dependiente del Servicio de Anestesiología, se ha traducido en la disminución de ingresos programados, liberando al SMI de muchos de sus compromisos en el mantenimiento del programa quirúrgico del centro. Por otra parte, se constata un aumento de la demanda de servicio en pacientes no quirúrgicos. ¿Es ello reflejo de una mayor actividad del centro, se autolimitaba la demanda en función de la programación quirúrgica, ha aumentado la complejidad de la asistencia global o se ha deteriorado la capacidad de respuesta de las plantillas médicas? En tanto y cuanto no exista una respuesta estructurada a estas preguntas, es evidente que la apertura de una nueva prestación (REA) no debe ir acompañada de una disminución de la capacidad asistencial del SMI (aquello de que ahora no será tan necesario disponer de tantas camas). El anunciado fenómeno del incremento de demanda de asistencia a pacientes críticos se cumple también en nuestro centro.

\section{Ejemplo B}

Disponibilidad de camas libres al inicio de la jornada. El análisis de los datos presentados (fig. 4) demuestra que la mínima disponibilidad corresponde al mismo periodo en que la cirugía programada condicionaba parte de la actividad. Las camas «libres»se concentraban a una hora tal que se pudiera absorber los ingresos procedentes del área quirúrgica. La disminución de este tipo de enfermos representa que los

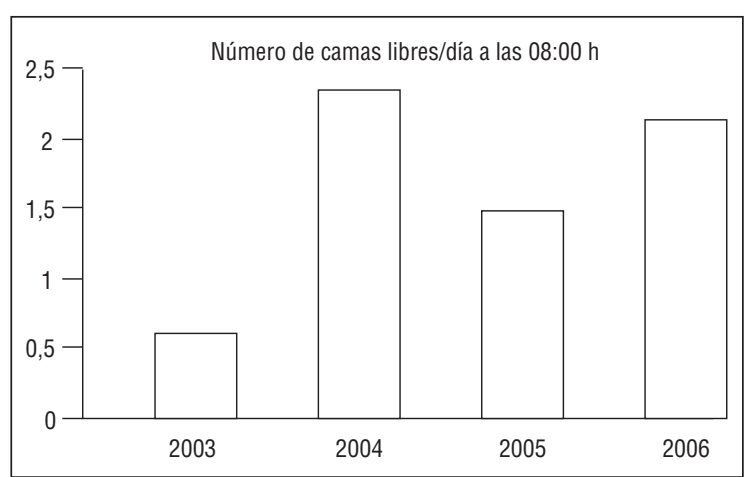

Figura 4. Número de camas libres (promedio anual) a las $8.00 \mathrm{~h}$ (datos propios).

flujos de pacientes no se producen a horas concretas, sino repartidos a lo largo del día, y por ello el número de camas desocupadas a la $8.00 \mathrm{~h}$ disminuye. Si esto se acompaña del mantenimiento del número de ingresos y de la estancia promedio, puede afirmarse que el uso de los recursos disponibles se realiza dentro de los parámetros deseados, e incluso recomendables.

\section{Ejemplo C}

La figura 5 ofrece una visión resumida de la necesidad de equipos de ventilación mecánica en función del porcentaje de pacientes ventilados. Se comprueba cómo el porcentaje de soportes ventilatorios realizados está entre el 40 y el $45 \%$. A ello hay que añadir la consideración del incremento de procedimientos ventilatorios no invasivos realizados en los últimos años. ¿Cuál puede ser la interpretación? Hay dos posibles. Una, es conveniente mantener el actual parque de aparatos de ventilación porque no se asiste a una disminución de las necesidades de los mismos, sino que, por contrario, es de prever que la tendencia detectada exigirá reconvertir algunos de los equipos en modelos más actuales con nuevas y mejores prestaciones, sobre todo en el campos de la ventilación no invasiva.

\section{Ejemplo D}

Una cuestión de máximo alcance y repercusión es la presencia de complicaciones infectivas, nosocomiales en su mayoría, que exige plantear cambios en los procedimientos de atención a los pacientes críticos. Concretamente, la instauración de medidas de barrera y el aislamiento de los casos confirmados o posibles de alta probabilidad. La figura 6 constata que, desde que se inició la recogida de este dato (alta prevalencia de infecciones por acinetobacter), las medidas instauradas se tradujeron en una disminución de la necesidad de aislamientos, y que en el momento en que el germen más prevalente ha virado (tanto en la UCI como en el resto del centro) a estafilococo se ha constatado un nuevo aumento de exi- 


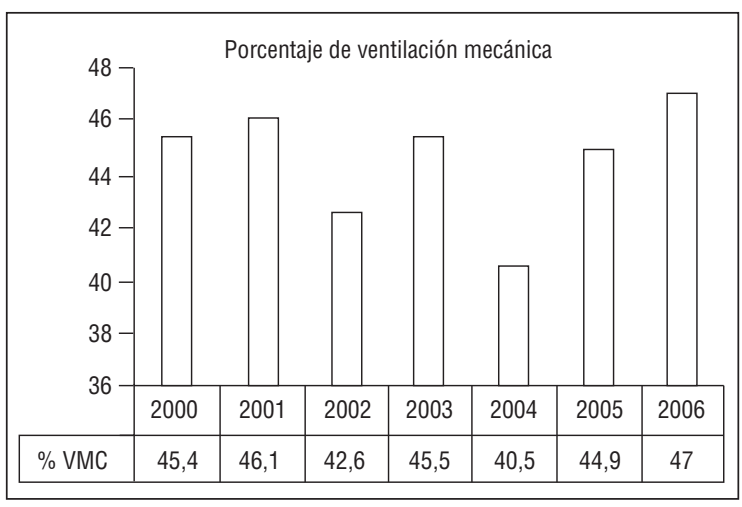

Figura 5. Porcentaje de enfermos ingresados/año sometidos a soporte ventilatorio (datos propios).

gencias de aislamiento que parecen volver a disminuir como consecuencia de un nuevo cambio en las políticas de aislamiento. La relación entre estas cifras de pacientes con altas necesidades de atención de enfermería y la dotación de personal de las UCI es evidente.

\section{SOBRE UN CONJUNTO MÍNIMO BÁSICO DE DATOS ESPECÍFICO DE SERVICIO O ESPECIALIDAD}

La evidencia de la necesidad de un sistema de información, centralizada, que supere la disponible a través de los CMBD convencionales, habitualmente gestionados por los Servicios de Admisión, pero que se escapen al detallismo individualizado de los PDMS y que sean, por tanto, más fácilmente interpretables y explotables en el Servicio, ha conducido a distintos autores (Ruiz en el Simposio de la SEMICYUC) ${ }^{14}$ a defender la existencia de un CMBD ampliado, propio de cada SMI, y cuya necesidad puede hacerse extensiva a cualquier otro Servicio asistencial.

Esto obliga a los proveedores informáticos a plantearse objetivos distintos, y más amplios, de los que se han contemplado hasta este momento. Aunque la mayoría de ellos aceptan una necesidad de "customizacion" o adaptación a la medida de las necesidades del cliente, lo cierto es que este hecho es más contemplado como concesión que como necesidad. No se trata de si uno u otro gestor clínico tiene el deseo de aumentar la complejidad de sus propios datos de información explotables, sino de comprender que la gestión clínica es algo distinto de la gestión de pacientes, que se mueve en otras coordenadas, con otras necesidades y otros planteamientos de base.

Cuando esto sea asumido por la industria de equipamientos ofimáticos y por los responsables informáticos de los centros, se habrá dado un gran paso adelante.

\section{RECOMENDACIÓN: AGÉNCIESE UN UDMS}

¿Y mientras? La respuesta a esta pregunta no es satisfactoria, ni siquiera animosa, sino algo agorera.

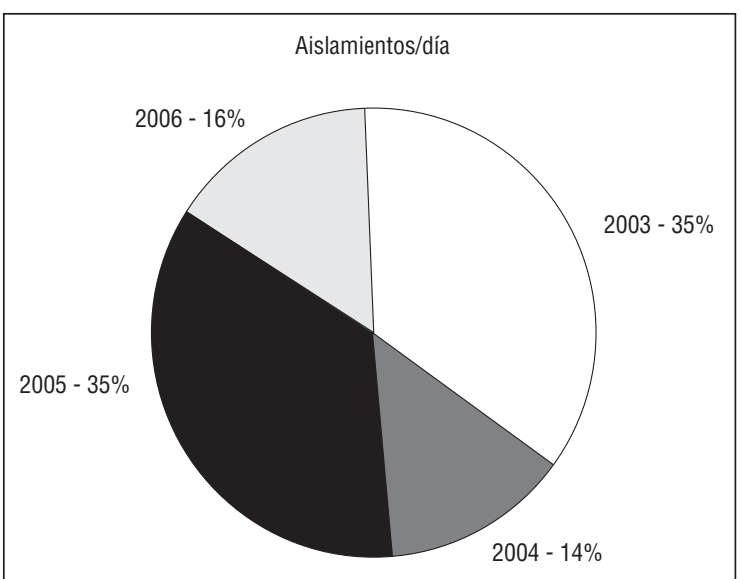

Figura 6. Número de pacientes/día sometidos a aislamiento y técnicas de barrera por la presencia de infecciones por gérmenes multirresistentes (datos propios).

Quien quiera disponer de la información que representa un UDMS tal y como lo hemos venido defendiendo hasta aquí debe estar dispuesto a realizar una ardua labor de «papel y lápiz». Gran parte de la información deseada, de los datos necesarios, que no puedan ser proporcionados desde los Servicios Centrales del hospital, tendrán que ser recogidos a mano, con la colaboración de secretarias, enfermería y todos y cada uno de los médicos del equipo. La disciplina necesaria para «construirse» un sistema de gestión de Unidad no puede imponerse. Todos los afectados, de forma directa o indirecta, deben ser conscientes de la importancia y la trascendencia del esfuerzo que se les demanda; deben poder participar de la interpretación y utilidad de la información, de que el esfuerzo asistencial que se les demanda al llenar cuadernillos de datos no es inútil, sino todo lo contrario.

En estas condiciones, el responsable de la gestión clínica del Servicio, de la Unidad, podrá enfrentarse a las frecuentes amenazas que nos asaltan y a los intentos intempestivos de modificar las cosas, sólo por modificar o amparándose en sesgos de interpretación de los indicadores de actividad. Suponemos que es caer en una burda paráfrasis de las campañas publicitarias, pero la conclusión debería ser: si puede permitirse un PDMS, adelante; pero ponga un UDMS en su vida, porque ese sí que es imprescindible.

\section{Declaración de conflicto de intereses}

Los autores han declarado no tener ningún conflicto de intereses.

\section{BIBLIOGRAFÍA}

1. Ruiz Moreno J. Información básica para la gestión de un SMI (presentación personal). En: Simposio: puesta al día de los sistemas de informatización en Medicina Intensiva. Congreso SEMICYUC 2007, Madrid, 3-6 junio 2007. 
2. Álvarez Nebreda CC. Glosario de términos para la Administración y Gestión de los Servicios Sanitarios. Madrid: Díaz de Santos; 1998.

3. Ruiz Moreno J, Martín Delgado MC. Fundamentos de gestión sanitaria en Medicina Intensiva. En: Gestión estratégica en Medicina Intensiva. Roca J, Ruiz J, editores. Barcelona: EdikaMed; 2006. p. 3-14

4. Ruiz Moreno J, Martín Delgado MC. Importancia de la incertidumbre en la gestión de los Servicios de Medicina Intensiva. En: Gestión estratégica en Medicina Intensiva. Roca J, Ruiz J, editores. Barcelona: EdikaMed; 2006. p. 163-172.

5. Spangenberg J, van Der Poel JHR, Iapichino G. Management control in the ICU. In: Management of Intensive Care. Guidelines for better use of resources. Miranda DR, Williams A, Loirat P, editors. Dordrecht (NL): Kluwer Academic Pub; 1990. p. $103-23$.

6. Groom DA, Harris JW. Evaluation and selection of systems for automating clinical Operations. Biomed Instrum Technol. 1990;24:173-85

7. Sakamoto N. Availability of software services for a hospital information system. Int J Med Inform. 1998;49:89-96.

8. Bosman RJ, Oudemane van Straaten HM, Zandstra DF. The use of intensive care information systems alters outcome predictions. Intensive Care Med. 1998;24:953-8.
9. Suistomaa M, Kari A, Ruokonen E, Takala J. Sampling causes bias in APACHE II and SAPS II scores. Intensive Care Med. 2000;26:1727-9.

10. Nicolás Arcelís JM. Diseño e implantación de un sistema de información para la gestión en una Unidad de Cuidados Intensivos. En: Gestión estratégica en Medicina Intensiva. Roca J, Ruiz J, editores. Barcelona: EdikaMed; 2006. p. 273-87.

11. Metnitz PGH, Moreno RP, Almeida E, Jordan B, Bauer P, Campos RA, et al. SAPS 3 from evaluation of the patient to evaluation of the Intensive Care Unit. Part 1: objectives, methods and cohort description. Intensive Care Med. 2005;31:1336-44. El material electrónico sumplementario puede obtenerse en: http://www. pubmedcentral.nih.gov/articlerender.fcgi?tool=pubmed\&pubmedid=16132893 (consultado en julio de 2007).

12. Baigorri González F. Filosofía de la gradación asistencial. En: Gestión estratégica en Medicina Intensiva. Roca J, Ruiz J, editores. Barcelona: EdikaMed; 2006. p 203-10.

13. Galdós Anuncibay P. La Medicina Intensiva fuera de la UCI (outreach services). En: Gestión estratégica en Medicina Intensiva. Roca J, Ruiz J, editores. Barcelona: EdikaMed; 2006. p. 221-35.

14. Ricart Conesa A. Propuesta de un conjunto mínimo básico de datos para los Servicios de Medicina Intensiva. En: Gestión estratégica en Medicina Intensiva. Roca J, Ruiz J, editores. Barcelona: EdikaMed; 2006. p. 85-103. 